\title{
Parabolized Stability Equation Models for Turbulent Jets and Their Radiated Sound*
}

\author{
Kristjan Gudmundsson† Tim Colonius ${ }^{\ddagger}$
}

\begin{abstract}
In this paper we present several refinements to a wave-packet model of sound generation from large-scale turbulence. We examine heated and unheated jets at Mach numbers of 0.5 and 0.9. Pressure fluctuations associated with large-scale structures are modeled with the Parabolized Stability Equations (PSE) for linear disturbances to the turbulent mean-flow. We show that PSE provides better agreement with near-field microphone-array data at low frequencies than previous models based on linear stability theory. We examine the extent to which microphone data is contaminated by fluctuations uncorrelated with largescale structures. By filtering out the uncorrelated fluctuations, via the proper orthogonal decomposition (POD), better agreement between data and theory is obtained.
\end{abstract}

\section{Introduction}

The accurate prediction of aerodynamic sound from high speed jets remains a challenging problem. Due to the complexity of the flow field, direct numerical simulations (DNS) are prohibitively expensive and limited to small-scale jets. By modelling the effects of small (sub-grid) scales, Large Eddy Simulations (LES) allow a significant reduction in resolution requirements. Bodony \& Lele ${ }^{1}$ performed a series of LES computations of heated and unheated jets with Reynolds numbers on the order of $10^{4}$ to $10^{5}$. They used a Kirchoff surface to obtain far-field predictions, finding the accuracy of the overall sound pressure level to depend strongly on the jet Mach number.

While LES is a cheaper alternative to DNS it is still a challenge to apply it to realistic flow configurations. In addition, it does not by itself provide understanding of, or handles for control of the underlying mechanisms of sound production by large-scale structures. Both DNS and LES are capable of computing the acoustic far-field over a large range of frequencies and radiation angles. However this is not necessary for every purpose as the peak radiation intensity occurs at aft-angles and low-frequencies. Modelling this component of sound via a lower order method would thus be a valuable complement to LES approaches.

Sound radiated at low frequencies and aft angles is associated with large-scale coherent structures evolving in the turbulent flow. These structures evolve from small disturbances that undergo amplification via the instabilities of the jet shear layer, ${ }^{2-4}$ rolling up into axisymmetric and helical rings ${ }^{5}$ before eventually loosing their coherence via non-linear interactions. The early development of large-scale coherent structures is largely linear, however. Several studies ${ }^{2,6,7}$ have shown the velocity and pressure fluctuations of forced jets to be well approximated by the eigenfunctions of the Orr-Sommerfeld equation, where the spatial stability problem is solved. The disturbances of natural jets are correlated over shorter times and distances, making their identification more difficult. However, Suzuki \& Colonius ${ }^{8}$ found good agreement near the most amplified frequency between pressure predictions of linear stability analysis and measurements from a phased microphone array, indicating that energy transfer between coherent and incoherent motions has only minor effects on the former, even for natural jets. Suzuki \& Colonius conducted their experiment in NASA Glenn's SHJAR facility; 78 microphones were placed on a conical surface just outside the jet shear layers in order

*Work supported by NAVAIR/TTC Technologies, Inc.

${ }^{\dagger}$ Ph.D. Candidate, Mechanical Engineering, California Institute of Technology, Pasadena, CA 91125, Student Member AIAA

$\ddagger$ Professor, Mechanical Engineering, California Institute of Technology, Pasadena, CA 91125, Senior Member AIAA 


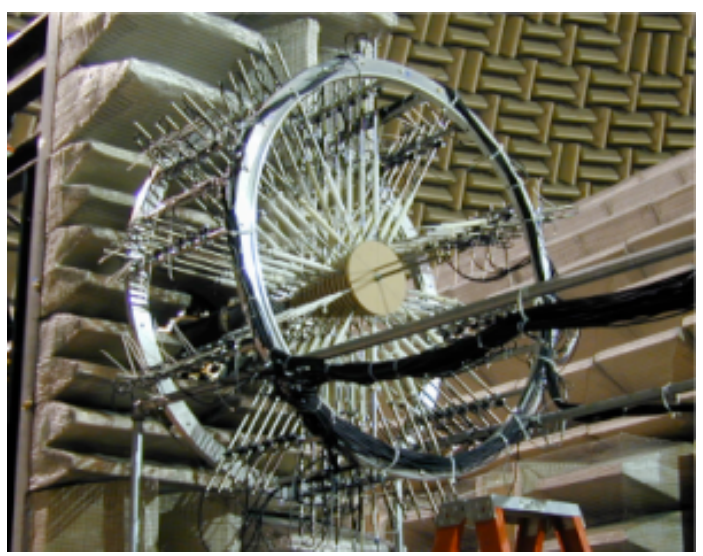

Figure 1. The Small Hot Jet Acoustic Rig (SHJAR) and Hydrodynamic Array at the NASA Glenn Research Center.

to resolve the azimuthal and streamwise variation of the pressure arising from instability waves. The same data is used in the present study. The microphone array is shown in figure 1. Instability wave envelopes computed for the turbulent mean-flow field (measured in the experiments with PIV) were directly compared to microphone data and indicated good agreement over a range of low frequencies (up to $S t=0.5$ ). They found poorer agreement for the axisymmetric $(m=0)$ mode at the lowest frequency considered $(S t=0.20)$ and attributed this to non-parallel effects. Presumably, these predictions would improve upon the explicit inclusion of non-parallel terms, and still further by the inclusion of non-linear interactions.

The Parabolized Stability Equations, introduced by Bertolotti \& Herbert, ${ }^{9,10}$ represent a refinement of the linear stability equations (LST) whereby both the non-parallel and non-linear effects can be retained in the analysis of slowly spreading, convectively unstable flows such as boundary layers, ${ }^{10-12}$ planar mixing layers ${ }^{13,14}$ and jets. ${ }^{15-18}$ The PSE have been used successfully to predict the far-field behavior of supersonic model jet flows, ${ }^{18}$ the mach wave radiation being well approximated by instability waves, provided their convection speed is supersonic. For subsonic jet flows the far-field predictions are not as good, but can be improved via projection methods, particularly for low angles, ${ }^{16,18,19}$ as the PSE are still able to model the near-field. Ryu et al. ${ }^{20}$ apply and extend the beamforming analysis of Suzuki \& Colonius ${ }^{8}$ to use instability waves computed with linear PSE (LPSE) using mean-flows from LES. They analyze two hot and cold supersonic cases in terms of near and far-field pressure. They also analyze a high-subsonic heated jet and find good agreement for azimuthal mode $m=1$ and frequencies $S t=0.1$ and 0.3. The authors attribute the unexpected mismatch of supersonic pressure predictions to non-linear contributions missing in their PSE simulation, as well as a potential contamination of the microphone data (from LES) due to Mach wave radiation.

The objective of the present work is to expand upon the work by Suzuki \& Colonius ${ }^{8}$ and Ryu et al. ${ }^{20}$ by employing both the LPSE and non-linear PSE (NPSE) in the investigation of the near and far-field sound characteristics of turbulent jets. Previous studies ${ }^{18,21}$ employing the NPSE in the simulation of jet flows have used mean-flows computed from the laminar flow equations, relying on the time average of the non-linear fluctuations (i.e., $F_{m 0}$ in equation ( 7 ) below) to alter the mean-flow as needed. This procedure is adequate for Reynolds numbers on the order of $10^{3}$, but fails to converge for significantly higher values. In this regime the mean-flow must be supplied from either independent simulations or time-averaged experimental measurements. In the present study we use mean-flows based on PIV measurements. We analyze two pairs of heated and cold subsonic jets and present comparisons between LPSE and LST to microphone measurements, as well as measurements filtered via the proper orthogonal decomposition (POD). 


\section{Computational Method}

We non-dimensionalize field variables as

$$
u=\frac{u *}{a_{\infty}} ; \quad \rho=\frac{\rho *}{\rho_{\infty}} ; \quad p=\frac{p *}{\rho_{\infty} a_{\infty}^{2}} ; \quad T=\frac{c_{p} T *}{a_{\infty}}
$$

where stars denote dimensional variables, $a_{\infty}$ and $\rho_{\infty}$ respectively denote ambient sound-speed and density, $c_{p}$ represents specific heat at constant pressure, and $u$ denotes any of $u_{x}, u_{r}$ or $u_{\theta}$. Spatial dimensions are normalized by jet diameter $D$. The governing equations, so scaled, are given by

$$
\begin{aligned}
\rho \frac{D u_{x}}{D t} & =-\frac{\partial p}{\partial x}+\frac{1}{R e} V_{x} \\
\rho\left(\frac{D u_{r}}{D t}-\frac{u_{\theta}^{2}}{r}\right) & =-\frac{\partial p}{\partial r}+\frac{1}{R e} V_{r} \\
\rho\left(\frac{D u_{\theta}}{D t}+\frac{u_{r} u_{\theta}}{r}\right) & =-\frac{1}{r} \frac{\partial p}{\partial \theta}+\frac{}{R e} V_{\theta} \\
\rho\left(\frac{D T}{D t}+(\gamma-1) T \nabla \cdot u\right) & =\frac{\gamma}{R e} \Phi+\frac{\gamma}{\operatorname{RePr} q} \\
\frac{D \rho}{D t}+\rho \nabla \cdot u & =0
\end{aligned}
$$

where $\nabla \cdot$ and $D / D t$ respectively denote the divergence operator and the convective derivative. The vector $V$ and scalars $\Phi$ and $q$ represent the viscous terms, viscous dissipation and heat conduction, respectively. $\gamma$ is the ratio of specific heats, taken as 1.4 in the current simulations. The Reynolds number is defined as $R e=\rho_{\infty} a_{\infty} D / \mu_{\infty}$ and the Prandtl number as $\operatorname{Pr}=\mu_{\infty} c_{p} / k_{\infty}$, set at 0.72 . Lastly, we assume ideal-gas behavior,

$$
p=\frac{\gamma-1}{\gamma} \rho T
$$

We decompose the solution $\phi(x, r, \theta, t)=\left[u_{x}, u_{r}, u_{\theta}, T, \rho\right]^{T}$ into its mean and fluctuating parts, such that

$$
\phi=\bar{\phi}+\phi^{\prime}
$$

where $\phi^{\prime}$ represents the fluctuations about the mean state, $\bar{\phi}(x, r)=\left[\bar{u}_{x}, \bar{u}_{r}, 0, \bar{T}, \bar{\rho}\right]^{T}$. Following traditional PSE analysis as introduced by Bertolotti \& Herbert, ${ }^{10}$ we further decompose the fluctuations $\phi^{\prime}$ into normal modes as

$$
\phi^{\prime}(x, r, \theta, t)=\sum_{n=-N}^{N} \sum_{m=-M}^{M} \epsilon_{m n} \hat{\phi}_{m n}(x, r) e^{i \int^{x} \alpha_{m n}(\xi) d \xi} e^{i(m \theta-n \omega t)},
$$

where the real and imaginary parts of $\alpha_{m n}$ denote axial wave number and growth rate, respectively; $m$ is the azimuthal wavenumber and $\omega$ is the angular frequency, while $\epsilon_{m n}$ represents the initial amplitude and phase of mode $(m, n)$.

Implicit in this decomposition is the assumption that the streamwise variation of the fluctuations $\phi^{\prime}$ can be separated into a slowly-varying shape function and a rapidly-varying wave component. This assumption represents the parabolization of the fluctuation equations and allows a marching solution, the $x$-coordinate becoming time-like. We note, however, that the resulting equations for the $\hat{\phi}_{m n}$ are not completely parabolic, as discussed by Li \& Malik. ${ }^{22,23}$ This results in constraints on the smallest allowable marching-step, $\Delta x$. To unambiguously separate flow development into the two components, we impose the following normalization on the shape-functions $\hat{\phi}_{m n},{ }^{10}$

$$
\int_{0}^{\infty} \hat{\phi}_{m n}^{*} \frac{\partial \hat{\phi}_{m n}}{\partial x} r d r=0
$$


where the star denotes the complex-conjugate. This constraint removes any exponential behavior from the shape functions $\hat{\phi}_{m n}$, ensuring their slow (algebraic) streamwise variation.

Substituting the two decompositions given by equations (4) and (5) into system (2), we obtain in symbolic form the system of equations governing the evolution of shape-functions $\hat{\phi}_{m n}$ :

$$
\left(A_{m n}+B\right) \hat{\phi}_{m n}+C \frac{\partial \hat{\phi}_{m n}}{\partial x}+D \frac{\partial \hat{\phi}_{m n}}{\partial r}+\frac{1}{R e} E \hat{\phi}_{m n}=\frac{F_{m n}\left(\phi^{\prime}\right)}{\epsilon_{m n}} e^{-i \int^{x} \alpha_{m n}(\xi) d \xi}
$$

where we have grouped terms linear in $\hat{\phi}_{m n}$ on the left-hand side. Setting the forcing vector $F_{m n}$ to zero we recover the linear PSE equations in cylindrical coordinates. Further, setting $\bar{\phi}_{x}=\bar{u}_{r}=0$ we recover the linear stability equations. This serves as a useful check of the LPSE implementation, as shown in figure 2.
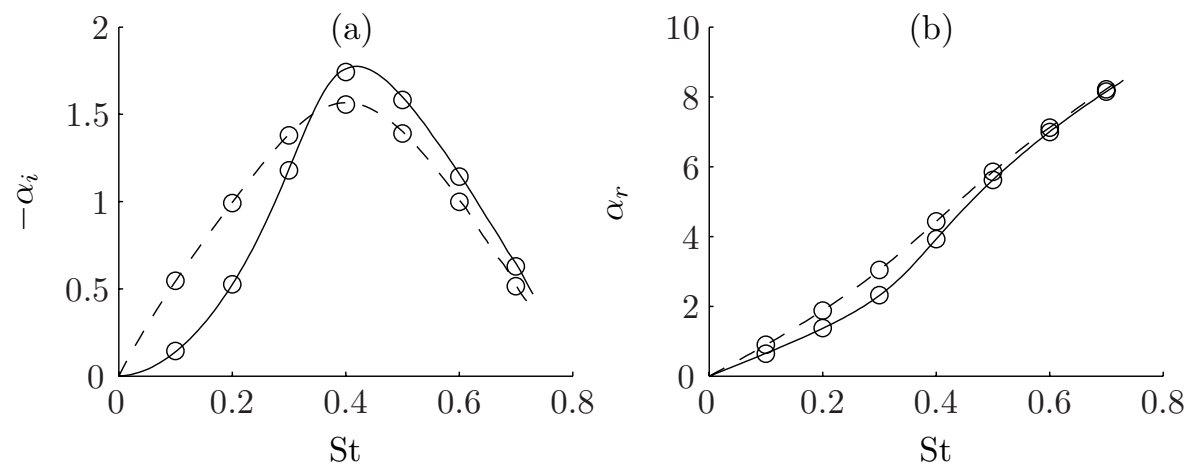

Figure 2. Spatial growth rates (a) and wavenumbers (b) obtained via linear stability analysis (-) and linear PSE with non-parallel terms omitted ( $($ ). Solid/dashed lines correspond to azimuthal modes $m=0 / 1$. Meanflow based on a tanh profile with $M_{\infty}=0.5$.

We discretize equation (7) using fourth-order central differences in the radial direction, and close the domain with the characteristic boundary conditions of Thompson. ${ }^{24}$ While previous studies ${ }^{25}$ have shown that boundary conditions based on asymptotic decay rates from linear stability analysis are sufficient, we find the characteristic formulation to be more robust, allowing a smaller computational domain. The streamwise derivative is approximated via first-order implicit Euler differences.

Initial conditions $\left(\hat{\phi}_{m n}, \alpha_{m n}\right)_{x_{0}}$ for equation (7) would optimally come from a solution which includes the local effects of flow spread. Day et al. ${ }^{13}$ used asymptotic expansions to this end, but found little benefit over the parallel-flow solution based on the initial profiles, $\bar{u}_{x}\left(x_{0}, r\right)$ and $\bar{\rho}\left(x_{0}, r\right)$. Upon setting $\bar{\phi}_{x}=\bar{u}_{r}=0$ (along with viscous terms), equation (7) can be reduced to the compressible Rayleigh equation,

$$
\frac{1}{r} \frac{d}{d r}\left(r \frac{d \hat{p}_{m n}}{d r}\right)-\left(\frac{2 \partial_{r} \bar{u}_{x}}{\alpha_{m n} \bar{u}_{x}-n \omega}+\frac{\partial_{r} \bar{\rho}}{\bar{\rho}}\right) \frac{d \hat{p}_{m n}}{d r}-\left(\frac{m^{2}}{r^{2}}+\alpha_{m n}^{2}-\bar{\rho}\left(\alpha_{m n} \bar{u}_{x}-n \omega\right)^{2}\right) \hat{p}_{m n}=0 .
$$

The Rayleigh equation is reduced to Bessel's equation near the axis and in the free-stream. The boundary conditions for the eigenfunctions $\hat{p}_{m n}$ follow from this;

$$
\text { as } r \rightarrow 0 \quad \hat{p}_{m n}(r) \sim J_{m}\left(\sqrt{\bar{\rho}\left(M_{\infty} \alpha_{m n}-n \omega\right)^{2}-\alpha_{m n}^{2}} r\right)
$$

and

$$
\text { as } r \rightarrow \infty \quad \hat{p}_{m n}(r) \sim H_{m}^{2}\left(\sqrt{n^{2} w^{2}-\alpha_{m n}^{2}} r\right),
$$

where $J_{m}$ and $H_{m}^{2}$ are the $m$-th order Bessel function of the first kind, and Hankel function of the second kind, respectively. The two-point boundary problem formed by equation (8) and boundary conditions (9-10) is solved via a shooting procedure. Using $\hat{p}_{m n}$, other components of $\hat{\phi}_{m n}\left(x_{0}, r\right)$ can be calculated directly. 
Before starting the marching procedure, we take the additional step of solving system (7) without nonparallel terms, but including viscous terms. This allows the linear stability solution to adjust to the effects of viscosity and reduces transients in the marching solution.

\section{Experimental Measurements}

We investigate two pairs of heated and cold subsonic jets at Mach numbers of 0.5 and 0.9 . The flow conditions are listed in table 1. Mean-flow data was obtained from stereoscopic PIV measurements conducted by Bridges \& Wernet $^{26}$ and Bridges \& Brown ${ }^{27}$ in the Small Hot Jet Acoustic Rig (SHJAR) at the NASA Glenn Research Center. To avoid numerical artifacts in the solution of equation (7) we smooth the PIV mean-flow by fitting it with a Gaussian function similar to that used by Tam \& Burton, ${ }^{28}$

$$
\bar{u}_{x}(x, r)= \begin{cases}M_{\infty} & \text { if } r<h(x) \\ M_{\infty} c(x) \exp \left(-\frac{(r-h(x))^{2}}{b(x)^{2}}\right) & \text { else, }\end{cases}
$$

where the functions $b(x), h(x)$ and $c(x)$ are determined via least-squares. Figure 3 shows an example of the fit so obtained for the heated Mach 0.5 jet. Similar fits can be obtained in the near-nozzle region with the hyperbolic-tangent function but this match deteriorates as the jet takes on a Gaussian shape further downstream. Temperature measurements were not available and were estimated from the Crocco-Busemann relation,

$$
\frac{T}{T_{\infty}}=-\frac{\bar{u}_{x}^{2}}{2}+\left(\frac{1}{\gamma-1}\left(\frac{T_{j e t}}{T_{\infty}}-1\right)+\frac{M_{\infty}^{2}}{2}\right) \frac{\bar{u}_{x}}{M_{\infty}}+\frac{1}{\gamma-1} .
$$

Transverse velocity profiles $\bar{u}_{r}$ were estimated from the mass-conservation equation.

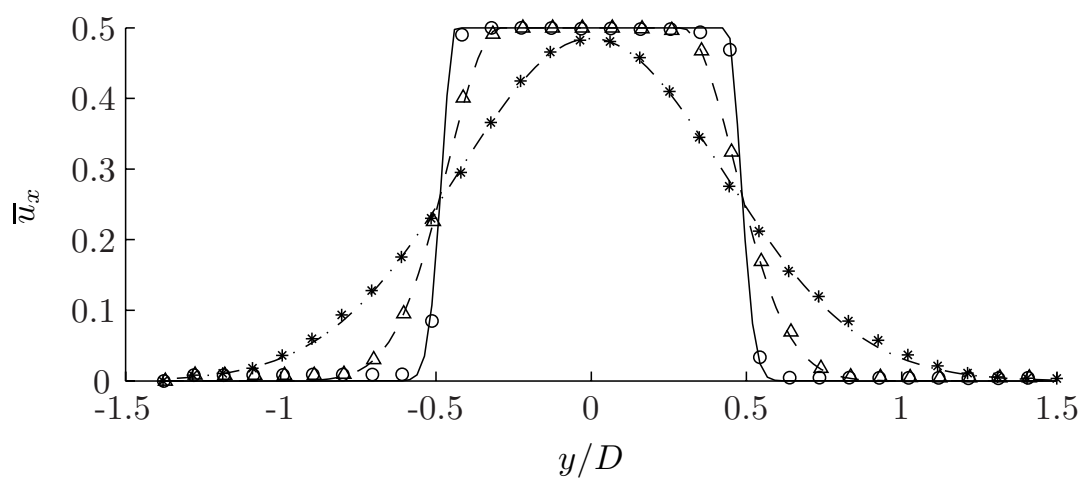

Figure 3. Fitting the PIV measurements (symbols) of the heated Mach 0.5 jet with the analytical function (lines) given by equation $(11)$ at axial stations $x / D=0.5(\circ ;-), 2.0(\triangle$; - $)$ ) and $5.0(* ;-\cdot-)$.

\begin{tabular}{cccc} 
Set Point & $M_{\infty}$ & $T_{j e t} / T_{\infty}$ & $R e=(\rho a D / \mu)_{\infty}$ \\
\hline 3 & 0.5 & 0.96 & $2 \cdot 10^{5}$ \\
23 & 0.5 & 1.76 & $5 \cdot 10^{4}$ \\
7 & 0.9 & 0.84 & $17 \cdot 10^{5}$ \\
27 & 0.9 & 1.76 & $2 \cdot 10^{5}$
\end{tabular}

Table 1. Flow conditions analyzed in this study. Set points are as defined by Tanna. ${ }^{29}$

Microphone measurements were obtained from the phased hydrodynamic-array (shown in figure 1) at the SHJAR. The array consists of thirteen concentric rings arranged on a conically expanding surface with the 
cone angle set to be slightly greater than the spread-angle of the flow. Each ring carries six equally-spaced microphones, allowing the accurate resolution of azimuthal modes $m=0$ and $m=1$. The radius of the array was chosen such as to place the microphones in the linear-hydrodynamic regime (see figure 4) where pressure fluctuations are largely hydrodynamic. An extensive review of the array design, experimental setup, data sampling and data processing may be found in Suzuki \& Colonius. ${ }^{8}$

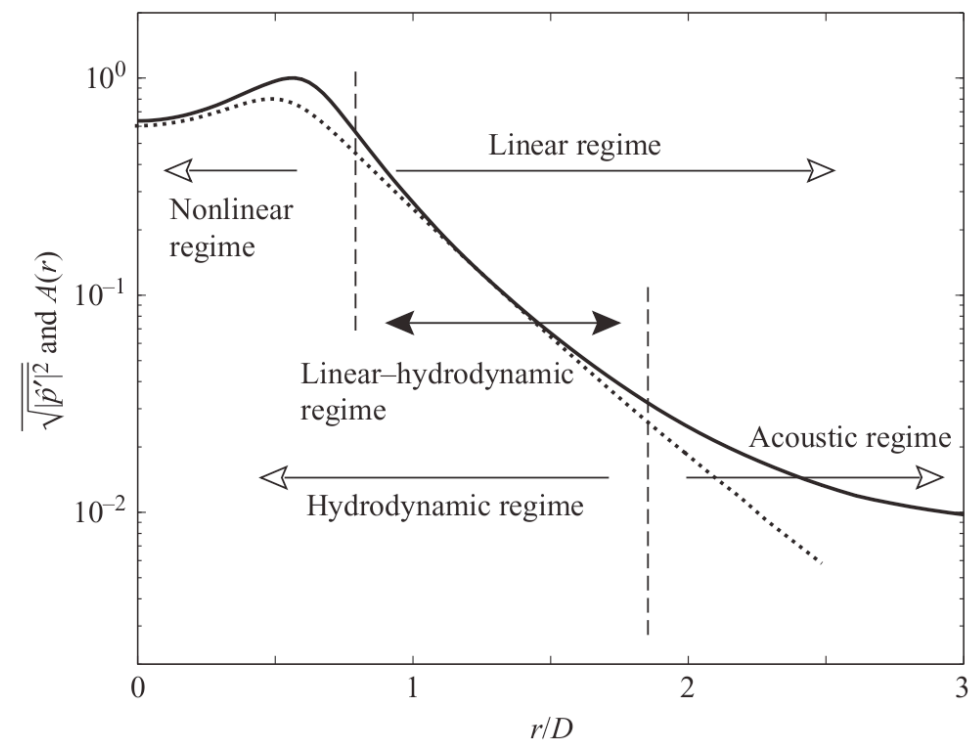

Figure 4. Root-mean-square pressure-fluctuations from $\operatorname{DNS}^{30}(-)$ compared with eigenfunction ( $\cdots$ ) from linear stability analysis for $m=0$ at $x / D=4$.

\section{Results and Discussion}

\section{A. Comparisons with Microphone Measurements}

Here we compare the predictions of LPSE with data from the phased array at NASA Glenn's SHJAR. We also include LST predictions for the cold Mach 0.5 jet. We solve the LST problem (equation (8)) at various axial-stations throughout the jet, using the local streamwise velocity $\bar{u}_{x}(x, r)$ and density $\bar{\rho}(x, r)$ profiles as input. To emulate the PSE expansion in equation (5) we construct a global LST solution, ${ }^{8}$

$$
\tilde{p}_{m n}(x, r)=\frac{\hat{p}_{m n}(r, \mid x)}{\max _{r}\left|\hat{p}_{m n}(r, \mid x)\right|} e^{i \int^{x} \alpha_{m n}(\xi) d \xi},
$$

where $\hat{p}_{m n}(r, \mid x)$ and $\alpha_{m n}(x)$ are respectively the local eigenfunction and eigenvalue for mode $(m, n)$, computed at axial station $x$.

Both the LST and LPSE solutions are independent of initial amplitude and phase (represented by $\epsilon_{m n}$ in equation (5)). Suzuki \& Colonius ${ }^{8}$ used a beam-forming procedure whereby $\epsilon_{m n}$ was chosen as that maximizing the phase-match between the microphone data and the LST prediction. We determine $\epsilon_{m n}$ via amplitude-matching, choosing $\epsilon_{m n}$ as that minimizing the error defined as

$$
J\left(\epsilon_{m n}\right)=\sum_{j=1}^{N_{m i c}}\left|\hat{P}_{m n}^{m i c}\left(x_{j}, r_{j}\right)-\epsilon_{m n} \tilde{p}_{m n}\left(x_{j}, r_{j}\right)\right|^{2},
$$

where $\hat{P}_{m n}^{m i c}\left(x_{j}, r_{j}\right)$ represents the processed microphone data on ring $j$, and $\tilde{p}_{m n}\left(x_{j}, r_{j}\right)$ represents the

$$
6 \text { of } 21
$$


LST/LPSE solution. This results in the estimate

$$
\epsilon_{m n}=\frac{\sum_{j=1}^{N_{m i c}}\left|\tilde{p}_{m n}\left(x_{j}, r_{j}\right) \hat{P}_{m n}^{m i c}\left(x_{j}, r_{j}\right)\right|}{\sum_{j=1}^{N_{m i c}}\left|\tilde{p}_{m n}\left(x_{j}, r_{j}\right)\right|^{2}} .
$$

Phase-matching is in general expected to return lower estimates of $\epsilon_{m n}$ as it attempts to filter out contributions from uncorrelated events (see section B below).

Figures 5 and 6 show the evolution of pressure amplitude and phase for the cold Mach 0.5 jet at $m=0$ and $m=1$, respectively, where the amplitudes $\epsilon_{m n}$ for both the LST and LPSE has been determined via the procedure described above. Note that the essential difference between the LST and LPSE is the explicit inclusion of non-parallel terms by the latter. These include the streamwise derivatives of $\bar{\phi}$, and the radial velocity $\bar{u}_{r}$. This has pronounced effects at the lowest frequencies considered $(S t=0.1-0.2)$, particularly for the axisymmetric $(m=0)$ mode, as seen in figure 5 . At higher frequencies the two methods agree closely in terms of amplitude evolution for both $m=0$ and $m=1$. Lower frequency instability-waves have higher wavelengths (lower wavenumbers $\alpha_{r}$; see figure 2) and so experience greater mean-flow variance over a single period. Conversely, the mean-flow looks increasingly parallel to disturbances of ever higher frequencies, explaining the improved performance of LST. In terms of phase the two methods give similar results with the agreement varying only weakly with frequency.

While the PSE include non-parallel terms, they are not fully general in that the PSE decomposition (equation (5)) assumes a slow streamwise variance of the mean-flow, where 'slow' should be taken as relative to the wavelength of a given disturbance. At $S t=0.1$, the axisymmetric LPSE mode in figure 5 has a wave-number of $\alpha_{r} D \approx 1$, giving it a wavelength of $\lambda \approx 2 \pi D$. At this flow-condition the decay of centerline velocity starts at $x \approx 6.4 D$; the flow-spread can not be considered small relative to this disturbance. The mean-flow sensitivity at low-frequencies affects the global (over all $x / D$ ) as well as the local LPSE solution. This explains the kink observed in the LPSE solutions at the lowest frequencies. For the cold Mach 0.5 jet, for example, the kink that occurs at around $x \approx 6.4 D$ is due to a discontinuity in the first derivative of the radius $h(x)$ in equation (11) where centerline velocity-decay starts.

The overall agreement between the microphone data and LPSE predictions at this flow condition is fairly good. The $m=0$ prediction saturates prematurely at the lowest frequencies. The converse is true at the highest frequencies, with the best fit obtained at $S t \approx 0.35$. The $m=1$ prediction agrees well for all frequencies. Note however the departure of amplitude predictions from the microphone data starting at $x / D \approx 4$ (depending on frequency), where disturbances have saturated. The same is true for the phase prediction. At the lower frequencies the phase is well-matched for both $m=0$ and $m=1$. As the disturbance frequency is increased there is an increasing mismatch, with the measured phase leveling off both near the nozzle and after saturation. This is due to acoustic contamination of the microphone data, discussed below.

Figures 7, 8 and 9 show microphone measurements and LPSE predictions for the heated Mach 0.5 jet, the cold Mach 0.9 jet and the heated Mach 0.9 jet, respectively. The LPSE predictions are of similar quality here as for the cold Mach 0.5 jet, at least during the growth-saturation phase. However, the microphone measurements appear even further contaminated for these cases, particularly for the higher-speed jets. This is evident from both the slow decay of measured pressure amplitude and the leveling of measured phase.

Ryu et $a l .{ }^{20}$ perform a similar analysis, using mean-flow and pressure data from LES. They set up probe-locations along two arrays, one closer to the axis than the other. They study a heated subsonic jet and two supersonic jets, and present results for the $m=1$ mode. They find good matches for the subsonic jet in the growth-saturation phase after which the predictions diverge similarly as in the current data.

The microphone data is comprised of both hydrodynamic and acoustic pressure fluctuations. The phasedarray was designed ${ }^{8}$ such as to place the microphones in the linear-hydrodynamic regime (see figure 4 ) where pressure fluctuations are largely hydrodynamic. However, the extent and location of this regime varies with the flow condition, while the angle of the phased-array is fixed. As an example, the array spreads relatively faster for the heated Mach 0.5 jet than the cold Mach 0.5 , while the radial decay of the respective eigenfunctions are similar. Accurate measurements are particularly difficult for the higher speed jets as fluctuations in the linear-hydrodynamic regime scale with $M_{\infty}^{2}$, while fluctuations in the acoustic regime 
scale with $M_{\infty}^{4}$. This narrows the radial extent of the hydrodynamic regime and means more microphones will be situated in the acoustic regime. However, the good fit obtained around the saturation region seems to indicate that the measured fluctuations are mostly hydrodynamic there.

\section{B. POD-filtering of Microphone Data}

Acoustic disturbances have supersonic phase-speeds and radiate symmetrically from their source while hydrodynamic disturbances have subsonic phase-speeds (in subsonic mean-flows) and any acoustic radiation from such wave-packets is directive. ${ }^{31}$ The phase-speed $c_{p}$ at frequency $\omega$ is defined as $c_{p}=\omega / \alpha_{r}$, where the wave-number $\alpha_{r}$ is the slope of the phase curves in figures 5 through 9. The LPSE phase-predictions match measurements fairly well around the saturation point. Further downstream the slope of the measured phase decreases, indicating phase-speeds well above those predicted by LPSE. In some cases, such as the $S t=0.5$ and $m=0$ mode of the cold Mach 0.5 jet, (figure 5) the measured phase curve is nearly horizontal and would give very large or infinite phase-speeds. These presumably correspond to acoustic wave-fronts propagating near-normally to the array.

It seems natural to decompose the pressure measurements on the basis of phase-speed. Tinney \& Jordan ${ }^{32}$ performed an experiment where the near-field pressure of three co-axial subsonic jets was measured with a phased-array similar to the one in the experiment of Suzuki \& Colonius, ${ }^{8}$ but with a significantly higher spatial resolution (48 microphones on a line array along with a movable ring carrying 15 microphones). Tinney \& Jordan Fourier-transform their data in time and azimuthal angle, but also in the axial coordinate, obtaining the wave-number spectrum $\alpha_{r}$. They then reconstruct the two components (propagating and non-propagating) in physical space. Such a decomposition would presumably shed some light on the current comparisons but would involve significant errors due to the limited axial resolution of the microphone data.

Another way to glean information from the microphone data is via correlation analysis, or proper orthogonal decomposition (POD).$^{33}$ For clarity of presentation we omit the subscript $m n$ (denoting azimuthal mode $m$ of frequency $n \omega$ ) from all variables below. We compute the spectral-density tensor

$$
R_{i j}^{k}=\hat{P}^{k *}\left(x_{i}, r_{i}\right) \hat{P}^{k}\left(x_{j}, r_{j}\right),
$$

where $i$ and $j$ range from 1 through $N_{\text {ring }}$, and $\hat{P}^{k}\left(x_{j}, r_{j}\right)$ is the transformed measurement in ensamble $k$. We then form the ensamble-averaged cross-spectral density matrix $R=\sum_{k} R^{k} / N_{\text {ens }}$ and solve the eigenvalue problem

$$
R \mathbf{q}=\lambda \mathbf{q}
$$

By construction, $R$ is Hermitian and positive-definite so that $\lambda_{j} \geq 0$ and eigenvectors corresponding to different eigenvalues are orthonormal. The $\lambda_{j}$ are ordered such that $\lambda_{j+1} \leq \lambda_{j}$. A faster rate of decay of the $\lambda_{j}$ series indicates a higher correlation or coherence in the data. This is because the POD-modes are uncorrelated, which follows from their orthonormality;

$$
\mathbf{q}_{i} \cdot \mathbf{q}_{j}= \begin{cases}1 & \text { if } i=j \\ 0 & \text { else }\end{cases}
$$

Figure 10 shows the eigenvalues (normalized with $\lambda_{1}$ ) computed for the four jets. As discussed in the previous section, the phased-array measurements seem to have higher acoustic content at higher frequencies, particularly for the higher-speed and heated jets. The decay rates observed in figure 10 are consistent with this, the data becoming increasingly complex with frequency and flow-speed/temperature.

The spectral-density tensor $R_{i j}$ can be reconstructed from the POD-modes,

$$
R_{i j}=\sum_{n=1}^{N_{\text {ring }}} \lambda_{n} \mathbf{q}_{i}(n)^{*} \mathbf{q}_{j}(n) .
$$

This decomposition allows the POD-filtering of the measured data where only the highest-energy mode is retained,

$$
\tilde{P}\left(x_{i}, r_{i}\right)=\sqrt{\lambda_{1}} \mathbf{q}_{1}\left(x_{i}, r_{i}\right) .
$$

$$
8 \text { of } 21
$$



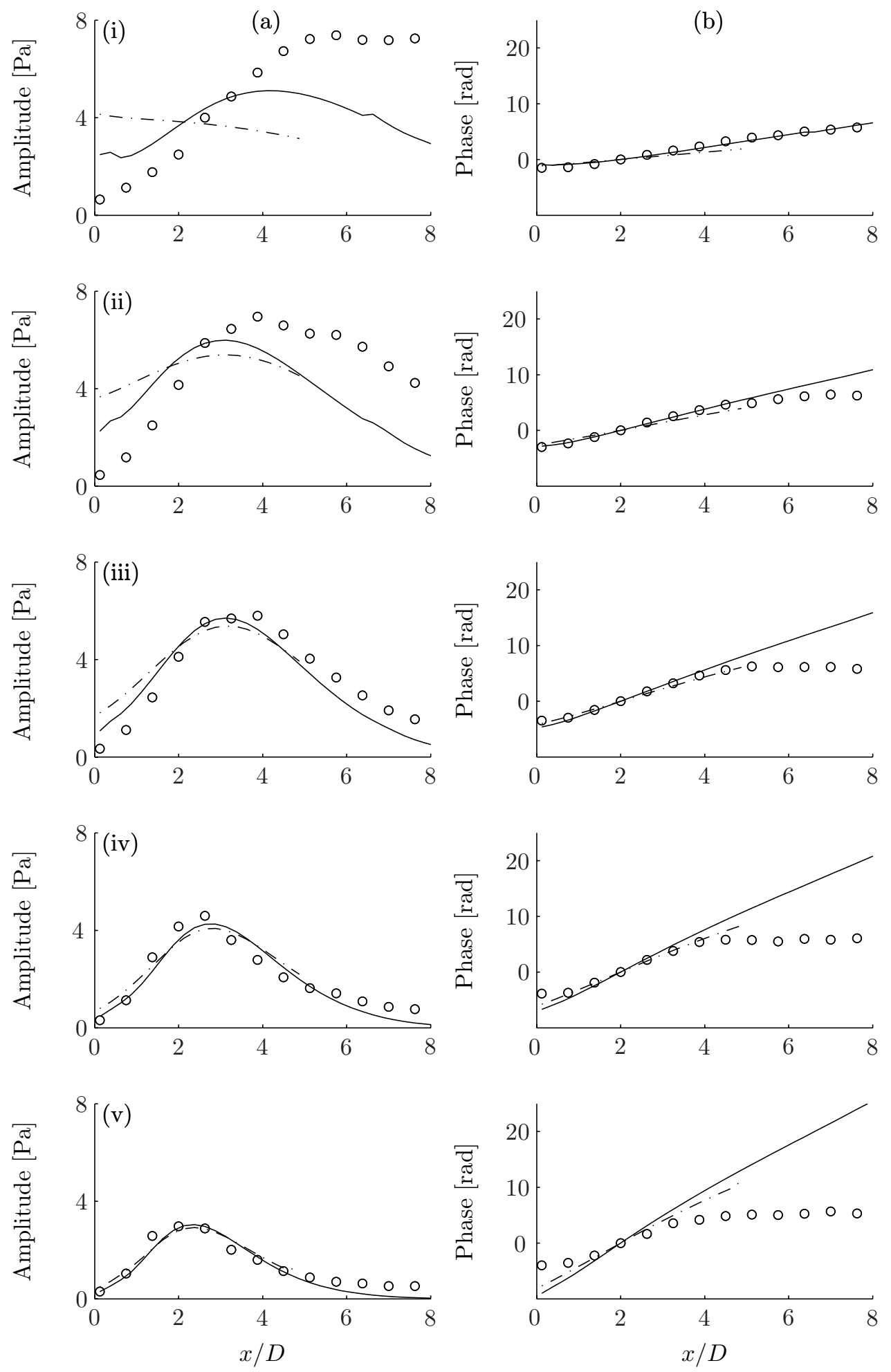

Figure 5. Evolution of pressure (a) amplitude and (b) phase along the phased-array for the axisymmetric mode $(m=0)$ of the cold Mach 0.5 jet. Microphone measurements denoted by symbols $(\circ)$ and predictions by lines (-, LPSE; ---, LST). Rows (i) through (v) show Strouhal numbers $S t=0.1$ through 0.5. 

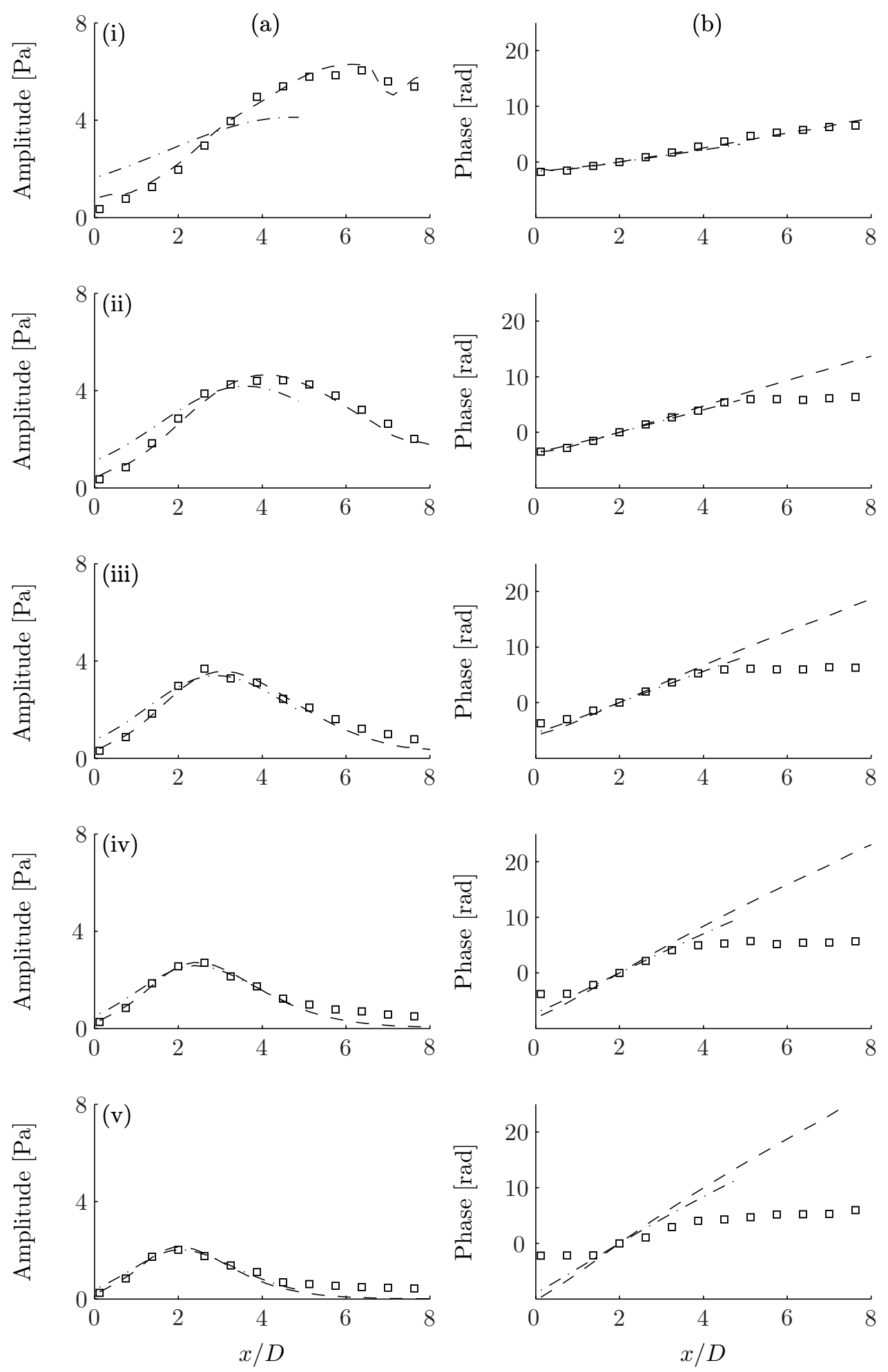

Figure 6. Evolution of pressure (a) amplitude and (b) phase along the phased-array for the first helical mode $(m=1)$ of the cold Mach 0.5 jet. Microphone measurements denoted by symbols $(\square)$ and predictions by lines $(--$, LPSE; ---, LST). Rows (i) through (v) show Strouhal numbers $S t=0.1$ through 0.5. 

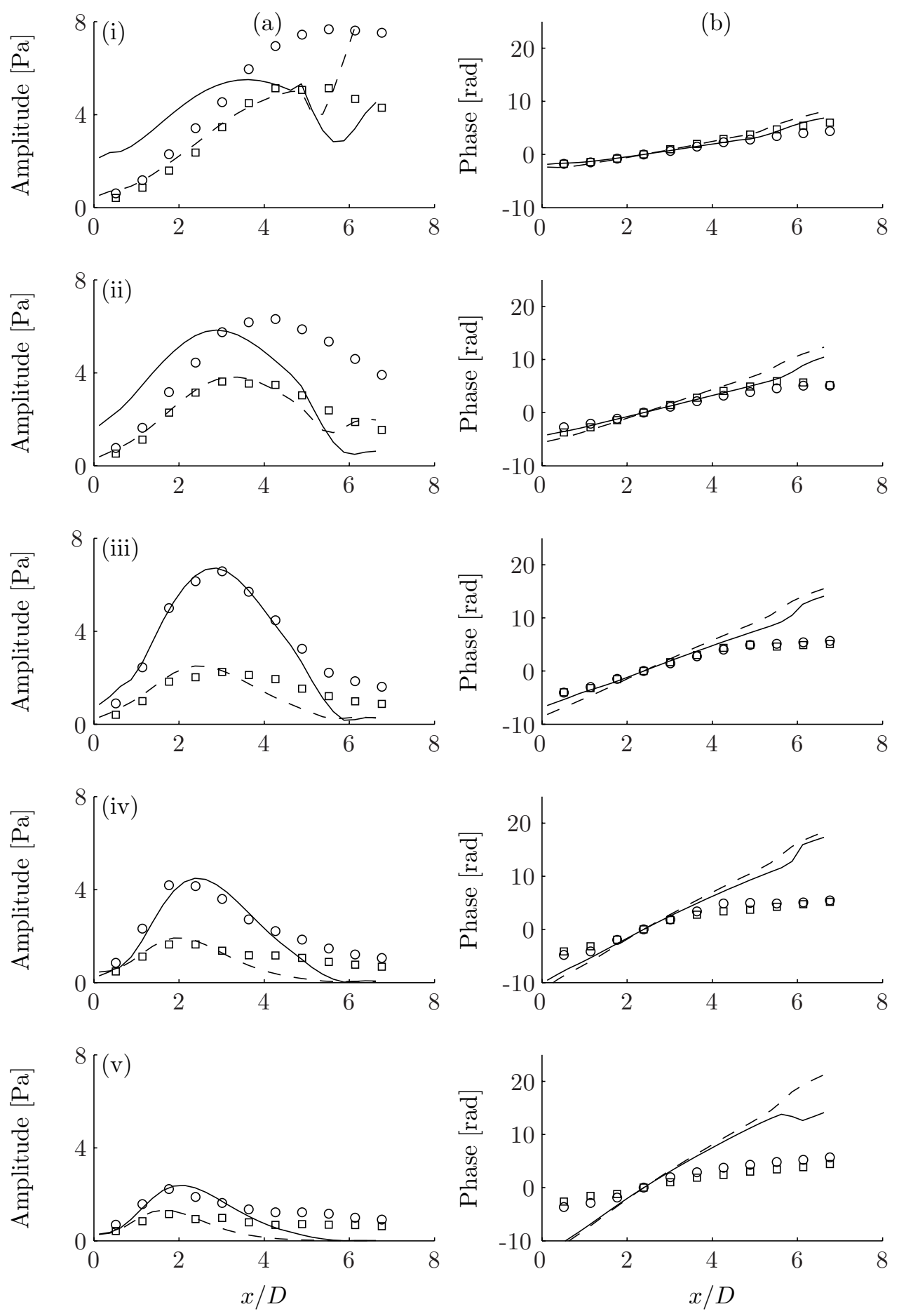

Figure 7. Evolution of pressure (a) amplitude and (b) phase along the phased-array for the heated Mach 0.5 jet. Microphone measurements denoted by symbols $(\circ, m=0 ; \square, m=1)$ and LPSE predictions by lines ($m=0 ;--, m=1$ ). Rows (i) through (v) show Strouhal numbers $S t=0.1$ through 0.5 . 

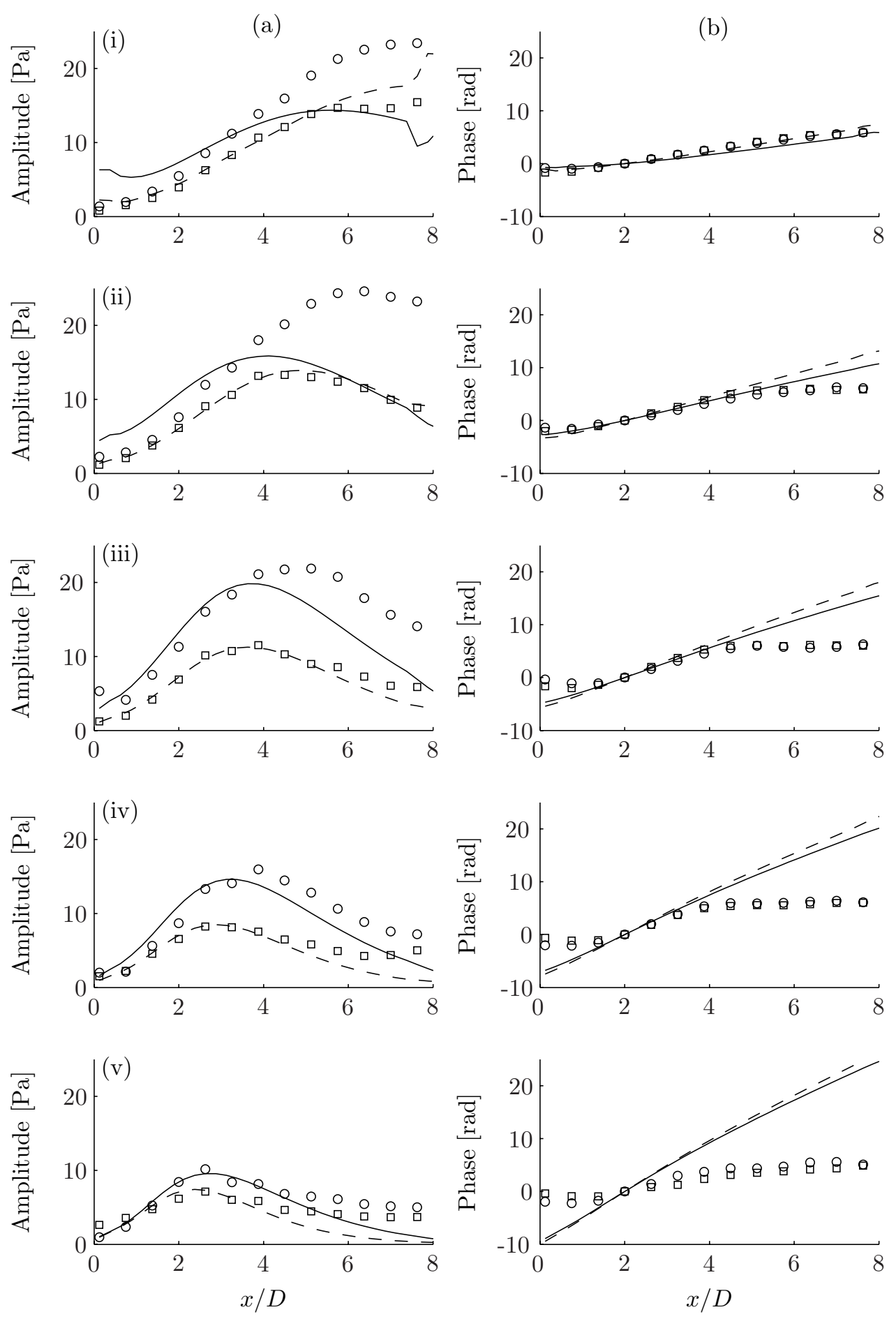

Figure 8. Evolution of pressure (a) amplitude and (b) phase along the phased-array for the cold Mach 0.9 jet. Microphone measurements denoted by symbols $(\circ, m=0 ; \square, m=1)$ and LPSE predictions by lines $(-, m=0$; $--, m=1)$. Rows (i) through (v) show Strouhal numbers $S t=0.1$ through 0.5 . 

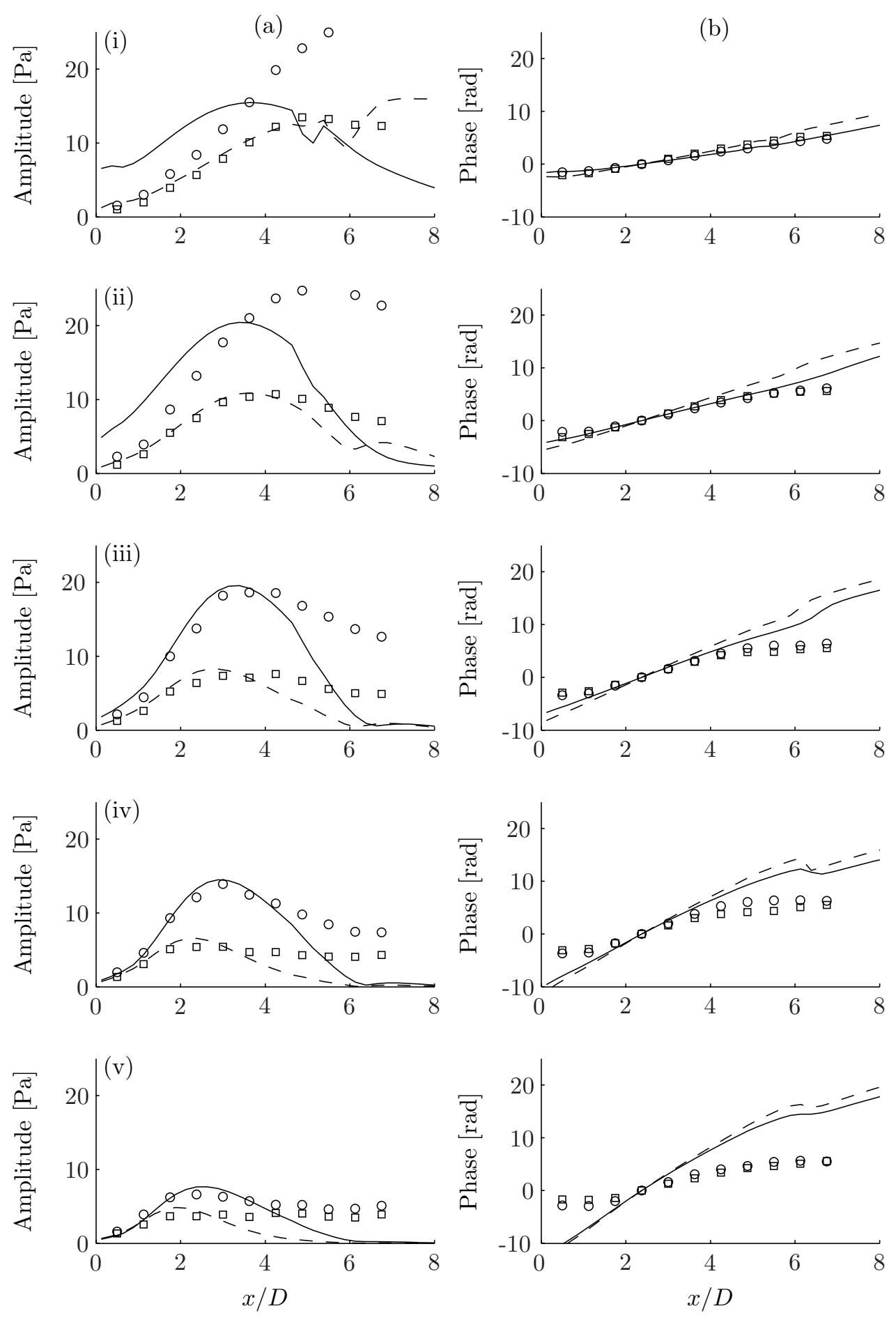

Figure 9. Evolution of pressure (a) amplitude and (b) phase along the phased-array for the heated Mach 0.9 jet. Microphone measurements denoted by symbols $(\circ, m=0 ; \square, m=1)$ and LPSE predictions by lines (-, $m=0 ;--, m=1)$. Rows (i) through (v) show Strouhal numbers $S t=0.1$ through 0.5. 
Figure 11 shows POD-filtered microphone measurements and LPSE predictions for the cold Mach 0.5 jet. While appearing the least contaminated, the measurements for this flow-condition do contain acoustic pressure, as evidenced by the slow decay observed after the saturation point as well as higher phase-speeds before and after the saturation point in figures 5 and 6 . The POD-filtering seems effective at removing acoustic components at this condition, as seen in the filtered phase relationship, which the LPSE prediction follows closely for both $m=0$ and $m=1$. Amplitude comparisons are improved as well, particularly for the higher frequencies where the LPSE predictions agree very well, even beyond the close of the potential core (at $x / D \approx 6.4)$.

Figures 12, 13 and 14 show the corresponding data for the heated Mach 0.5 jet, the cold Mach 0.9 jet and the heated Mach 0.9 jet, respectively. Similar agreement is obtained for the cold Mach 0.9 jet as for the cold Mach 0.5 jet but the relative improvement is more substantial at high speed where acoustic contamination of the microphone data is more significant. The same is true for the heated jets although not at the highest frequencies considered. For the heated Mach 0.5 jet, improvements are seen near the nozzle (mostly in terms of phase), but roughly a diameter after saturation, phase-speeds increase and the amplitude grows slightly again. This is also seen for the heated Mach 0.9 jet, where the POD-filtered amplitudes saturate at similar levels as for the unfiltered data. The $m=1$ POD-mode at $S t=0.5$ for this case starts out and remains of low amplitude, gradually rising around $x / D=3$ while its phase-speed remains higher than the LPSE prediction throughout, perhaps suggesting that this POD-mode is of acoustic origin. Figure 15 shows a close-up of this mode. Shown are the LPSE prediction $\hat{p}_{m n}$, microphone measurement $\hat{P}_{m n}$ and the two POD-modes $\tilde{P}_{1}$ and $\tilde{P}_{2}$ at $m=1$ and $S t=0.5$. The second POD-mode appears to be the one most akin to the hydrodynamic fluctuations predicted by LPSE. The LPSE prediction follows the early $(x / D \leq 2)$ amplitude development of both the microphone measurements and the second POD-mode. After this point only the second POD-modes approximates the LPSE behavior of saturation and decay. Similarly, the phase of LPSE prediction closely follows that of the second POD-mode, while both the measured phase and that of the first POD-mode represent higher phase-speeds.

\section{Conclusions and Future Work}

In this work we have solved the linearized stability equations and the linear parabolized stability equations in cylindrical coordinates. Using mean-flows based on time-averaged PIV measurements, ${ }^{26,27}$ we compute the linear near-field fluctuations of two pairs of heated and cold Mach 0.5 and 0.9 jets. We compare our predictions to near-field microphone measurements ${ }^{8}$ performed using the phased-array at NASA Glenn's SHJAR facility.

The overall agreement between the microphone data and LPSE predictions is good, the quality of fit depending on the flow condition, disturbance frequency $S t$ and azimuthal wave-number $m$. At the lowest frequency considered, the $m=0$ mode suffers from non-parallel effects (the LPSE require slow mean-flow spread; see discussion in section A), although the prediction is greatly improved from that of LST. This mode tends to saturate prematurely at the lowest frequencies, while the converse is true at the highest frequencies, with the best fit obtained at $S t \approx 0.35$. The $m=1$ prediction agrees well for all frequencies. Some acoustic contamination is observed in the data for all flow conditions. This is apparent from the slow amplitude decay and increasing phase-speeds downstream of the saturation point. Similar effects are observed in the near-nozzle region, although mostly in terms of phase-speed. These are particularly noticeable for the heated Mach 0.5 jet, and the two higher speed jets.

The microphone data is comprised of both hydrodynamic and acoustic pressure fluctuations. Proper Orthogonal Decomposition ${ }^{33}$ (POD) allows the separation of the data into uncorrelated components. We perform this analysis and compare LPSE predictions to the most energetic POD-mode. The comparisons so obtained are greatly improved, particularly for the higher-speed and heated jets which had appeared the most contaminated (compare figures 5-9 with figures 11-14). For the cold jets the LPSE predictions match very well, even beyond the end of the potential core. At the highest frequency considered, the acoustic disturbances in the heated-jet measurements have energies comparable to that of the hydrodynamic disturbances. For the heated Mach 0.9 jet, the acoustic energy at $S t=0.5$ is higher, the corresponding 

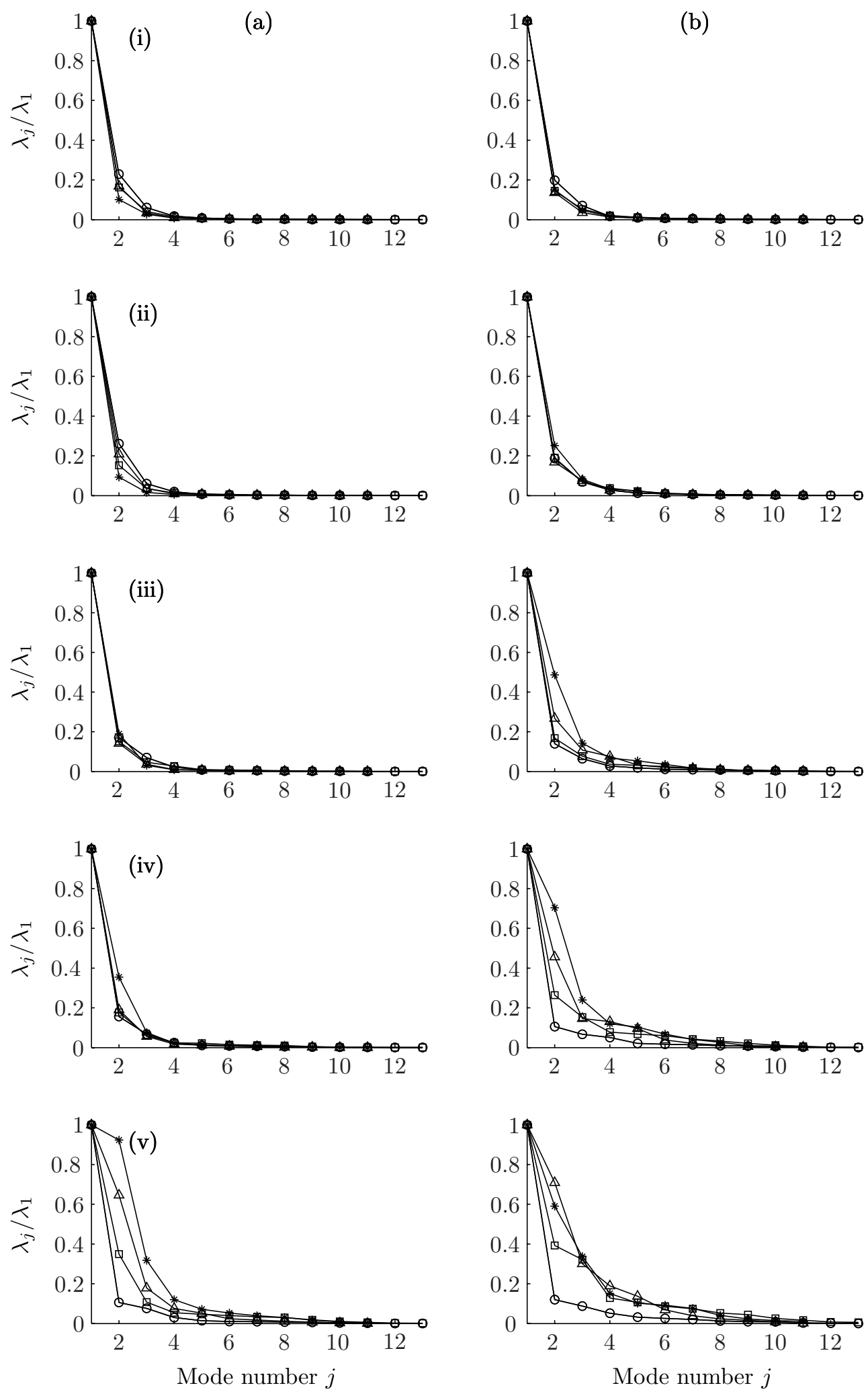

Figure 10. Normalized eigenvalues $\lambda_{j} / \lambda_{1}$ of the cross-spectral matrix (POD eigenvalues) for the (a) $m=0$ and (b) $m=1$ modes, at frequencies (i) $S t=0.1$ through (v) $S t=0.5$. Shown are the four flow conditions in this study ( $\circ$, cold Mach 0.5; $\square$, heated Mach 0.5; $\triangle$, cold Mach 0.9; *, heated Mach 0.9) 

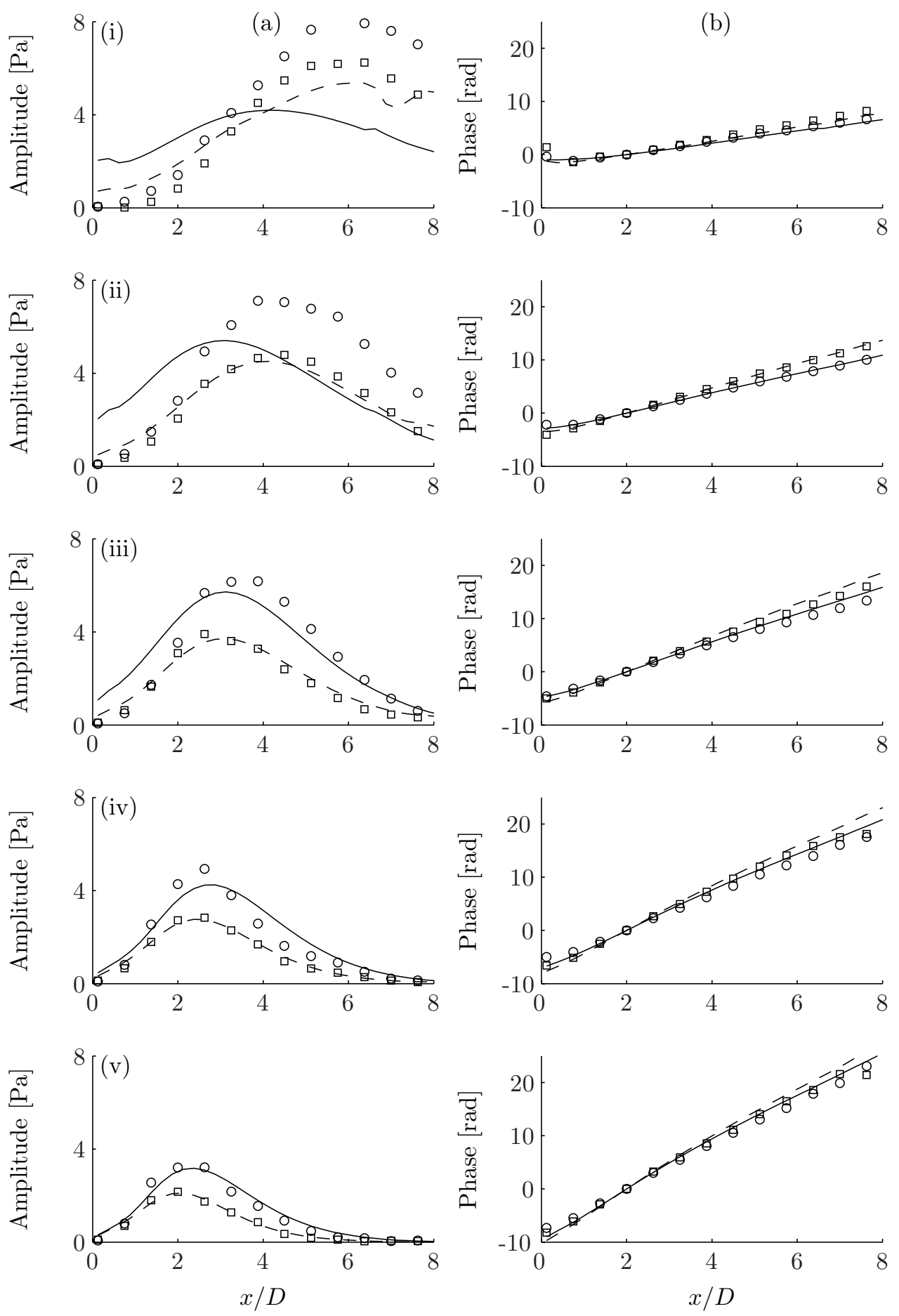

Figure 11. Evolution of pressure (a) amplitude and (b) phase along the phased-array for the cold Mach 0.5 jet. First POD-mode (equation (20)) denoted by symbols $(\circ, m=0 ; \square, m=1)$ and LPSE predictions by lines $(-, m=0 ;--, m=1)$. Rows (i) through (v) show Strouhal numbers $S t=0.1$ through 0.5 . 

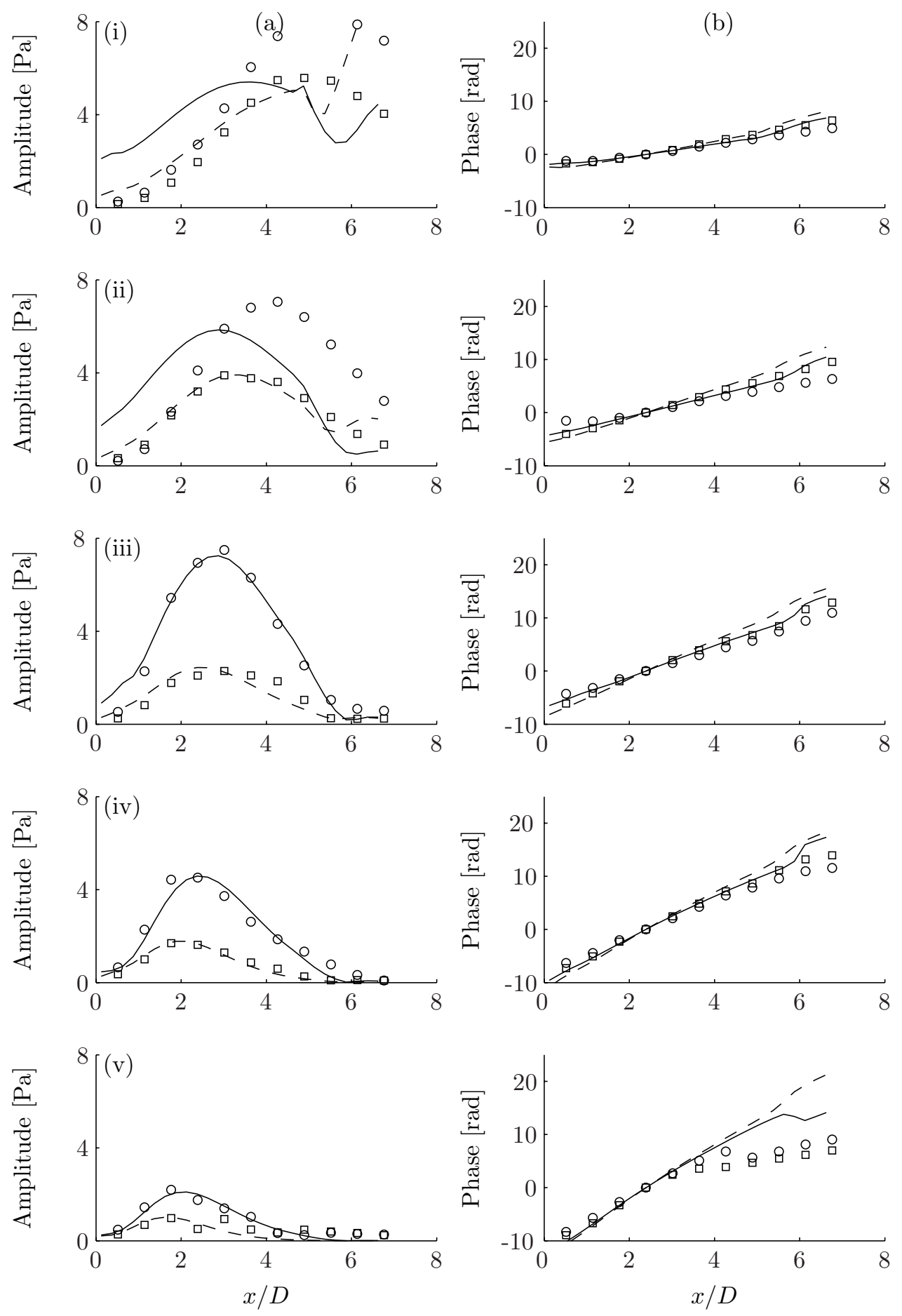

Figure 12. Evolution of pressure (a) amplitude and (b) phase along the phased-array for the heated Mach 0.5 jet. First POD-mode denoted by symbols $(\circ, m=0 ; \square, m=1)$ and LPSE predictions by lines $(-, m=0 ;--$, $m=1$ ). Rows (i) through (v) show Strouhal numbers $S t=0.1$ through 0.5 . 

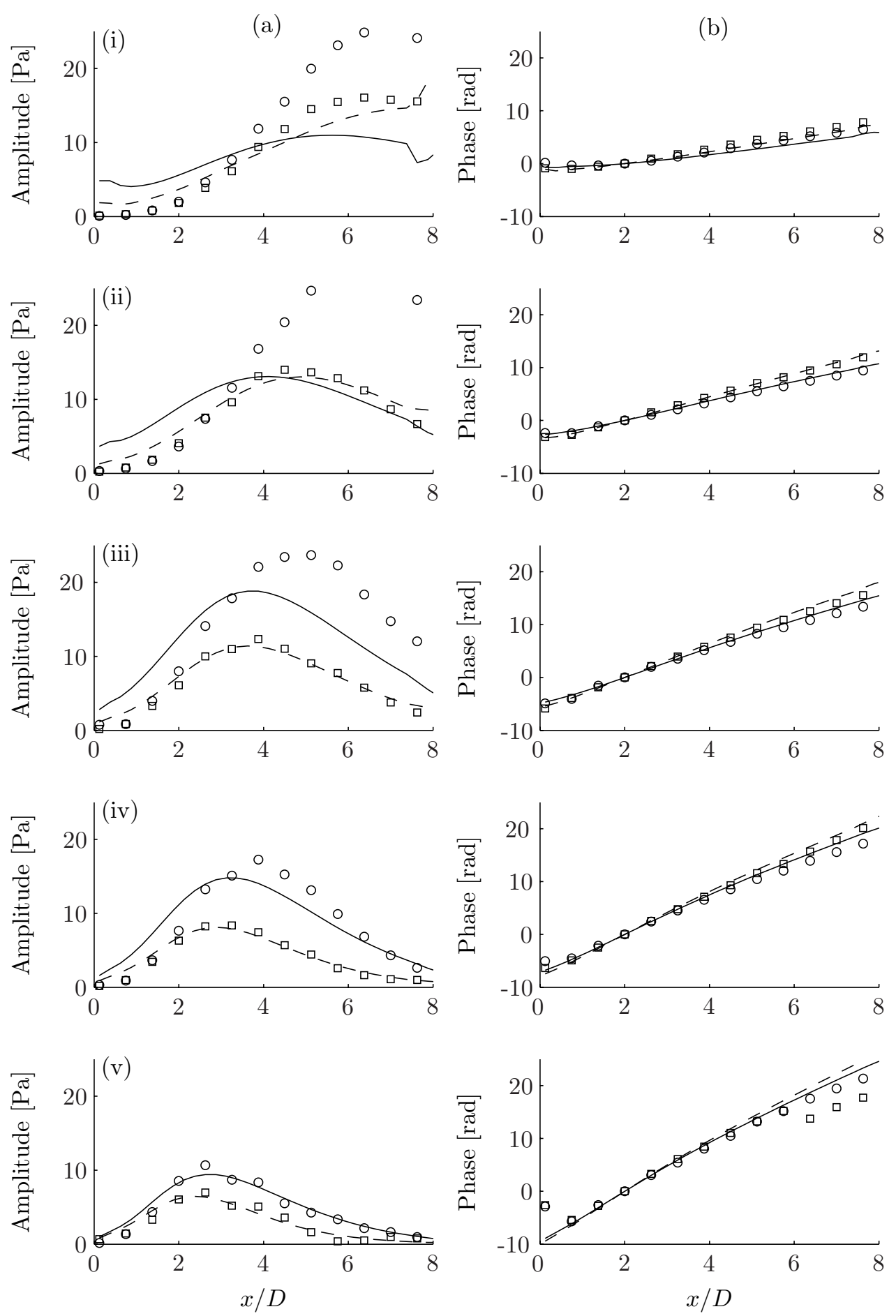

Figure 13. Evolution of pressure (a) amplitude and (b) phase along the phased-array for the cold Mach 0.9 jet. First POD-mode denoted by symbols $(\circ, m=0 ; \square, m=1)$ and LPSE predictions by lines $(-, m=0 ;--$, $m=1$ ). Rows (i) through (v) show Strouhal numbers $S t=0.1$ through 0.5 . 

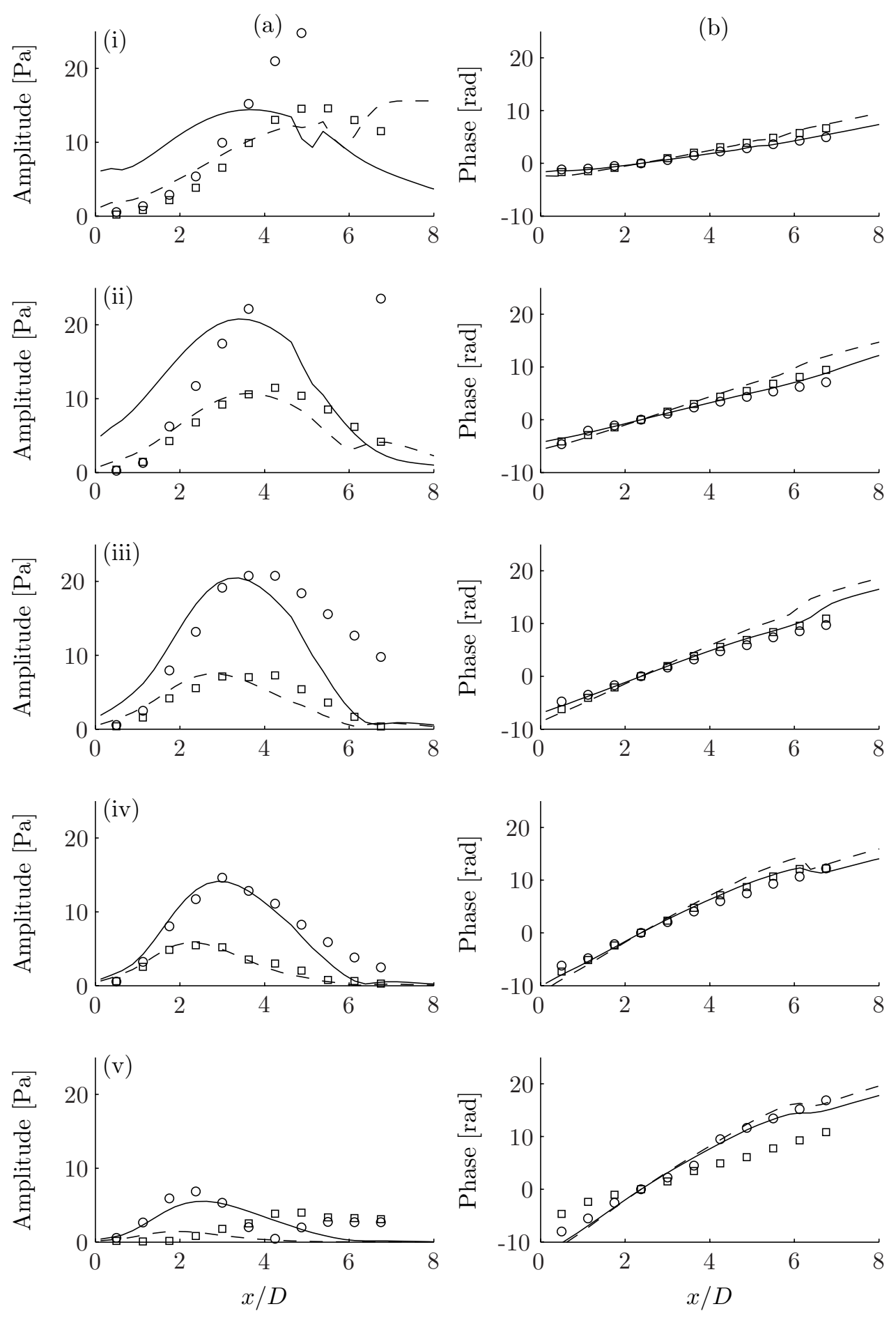

Figure 14. Evolution of pressure (a) amplitude and (b) phase along the phased-array for the heated Mach 0.9 jet. First POD-mode denoted by symbols $(\circ, m=0 ; \square, m=1)$ and LPSE predictions by lines $(-, m=0 ;--$, $m=1$ ). Rows (i) through (v) show Strouhal numbers $S t=0.1$ through 0.5 . 

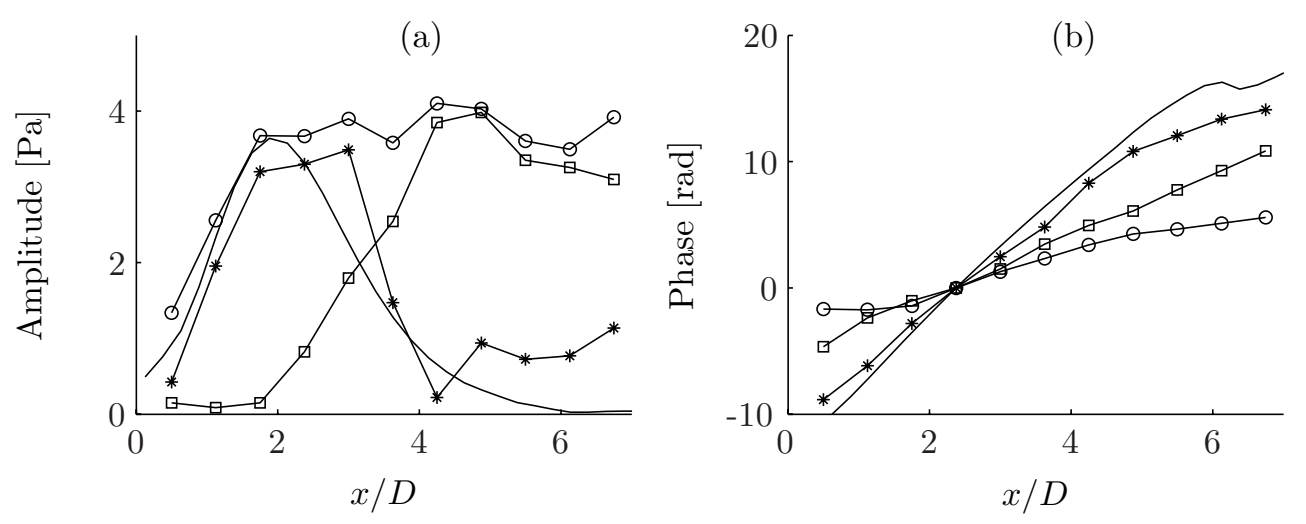

Figure 15. Evolution of pressure (a) amplitude and (b) phase along the phased-array for the heated Mach 0.9 jet, for $m=1$ and $S t=0.5$. Shown are the LPSE prediction $(-)$, microphone measurements (o), and the first $(\square)$ and second $(*)$ POD-modes.

LPSE prediction matching better with the second POD-mode than the first, as shown in figure 15.

While there remain some discrepancies between data and linear PSE, particularly for the axisymmetric mode at the lowest frequencies, PSE seems to provide an accurate representation of the streamwise evolution of pressure fluctuations well past the close of the potential core. It is possible, for example, that the inclusion of nonlinear effects will result in better agreement, and this is presently being pursued. It remains to compare the directly measured far-field sound by what would be implied by the near field fluctuations from PSE. PSE does not directly provide the acoustic far field (especially at subsonic speeds), but acoustic analogy and Kirchhoff-surface type approaches have been developed for this purpose. ${ }^{14,16,18,19,34}$ In particular, Reba et al. ${ }^{34}$ have successfully developed a Kirchhoff-surface approach and applied it to predicting sound from the same near-field microphone array data used in the present study. We plan to use a similar approach to project the current PSE predictions to the far-field.

\section{Acknowledgements}

This work was supported by NAVAIR/TTC Technologies. We would like to express great appreciation to Dr. James Bridges and colleagues at the NASA Glenn Research Center for providing us with their data, as well as to Dr. Takao Suzuki for help with data processing.

\section{References}

${ }^{1}$ Bodony, D. J. and Lele, S. K., "Using Large Eddy Simulation for Jet Noise Prediction," PF, Vol. 17, 2005.

${ }^{2}$ Mattingly, G. E. and Chang, C. C., "Unstable waves on an axisymmetric jet column," J. Fluid Mech., Vol. 63(3), 1974, pp. 541-560.

${ }^{3}$ Crighton, D. G. and Gaster, M., "Stability of Slowly Diverging Jet Flow," J. Fluid Mech., Vol. 77, 1976, pp. 397-413.

${ }^{4}$ Mankbadi, R. and Liu, J. T. C., "A Study of the Interactions Between Large-Scale Coherent Structures and Fine-Grained Turbulence in a Round Jet," Proc. Roy. Soc. London, Vol. 1443, 1981, pp. 541-602.

${ }^{5}$ Crow, S. C. and Champagne, F. H., "Orderly structures in turbulence," J. Fluid Mech., Vol. 48, 1971, pp. 547-591.

${ }^{6}$ Zaman, K. B. M. Q., Reeder, M. F., and Hussain, A. K. M. F., "Vortex pairing in a circular jet under controlled excitation. Part 1. General jet response," J. Fluid Mech., Vol. 101(3), 1980, pp. 449-491.

${ }^{7}$ Petersen, R. A. and Samet, M. M., "On the preferred mode of jet instability," J. Fluid Mech., Vol. 194, 1988, pp. 153-173.

${ }^{8}$ Suzuki, T. and Colonius, T., "Instability waves in a subsonic round jet detected using a near-field phased microphone array," J. Fluid Mech., Vol. 565, 2006, pp. 197-226.

${ }^{9}$ Herbert, T. and Bertolotti, F., "Stability Analysis of Nonparallel Boundary Layers," Bulletin of the American Physical Society, Vol. 32, 1987, pp. 2079. 
${ }^{10}$ Bertolotti, F. P. and Herbert, T., "Analysis of the Linear Stability of Compressible Boundary Layers using the PSE," Theoret. Comput. Fluid Dynamics, Vol. 3, 1991, pp. 117-124.

${ }^{11}$ Bertolotti, F. P., Herbert, T., and Spalart, P. R., "Linear and Nonlinear Stability of the Blasius Boundary Layer," J. Fluid Mech., Vol. 242, 1992, pp. 441-474.

${ }^{12}$ Chang, C. L., Malik, M. R., Erlebacher, G., and Hussaini, M. Y., "Linear and Nonlinear PSE For Compressible Boundary Layers," Tech. Rep. ICASE 93-70, 1993.

${ }^{13}$ Day, M. J., Mansour, N. N., and Reynolds, W. C., "Nonlinear stability and structure of compressible reacting mixing layers," J. Fluid Mech., Vol. 446, 2001, pp. 375-408.

${ }^{14}$ Cheung, L., Bodony, D. J., and Lele, S. K., "Noise radiation predictions from jet instability waves using a hybrid nonlinear PSE-acoustic analogy approach," AIAA Paper 2007-3638, 2004.

${ }^{15}$ Malik, M. R. and Chang, C. L., "PSE Applied to Supersonic Jet Instability," AIAA Paper 1997-0758, 1997.

${ }^{16}$ Balakumar, P., "Prediction of Supersonic Jet Noise," AIAA Paper 1998-1057, 1998.

${ }^{17}$ Yen, C. C. and Messersmith, N. L., "Application of Parabolized Stability Equations to Prediction of Jet Instabilities," AIAA J., Vol. 36, 1998, pp. 1541-1544.

${ }^{18}$ Cheung, L. and Lele, S. K., "Acoustic Radiation from Subsonic and Supersonic Mixing Layers with Nonlinear PSE," AIAA Paper 2001-0744, 2007.

${ }^{19}$ Lin, R., Reba, R. A., Narayanan, S., Hariharan, N. S., and Bertolotti, F. P., "Parabolized Stability Equation Based Analysis of Noise from an Axisymmetric Hot Jet," ASME HT-FED2004-56820, 2004.

${ }^{20}$ Ryu, J., Lele, S. K., and Viswanathan, K., "Investigation of the role of instability waves in jet noise radiation," AIAA Paper 2008-0026, 2008.

${ }^{21}$ Malik, M. R. and Chang, C. L., "Nonparallel and nonlinear stability of supersonic jet flow," Vol. 29, 2000, pp. 327-365.

${ }^{22} \mathrm{Li}$, F. and Malik, M. R., "On the Nature of the PSE Approximation," Theoret. Comput. Fluid Dynamics, Vol. 8, 1996, pp. 253-273.

${ }^{23}$ Li, F. and Malik, M. R., "Spectral Analysis of Parabolized Stability Equations," Vol. 3, 1997, pp. $279-297$.

${ }^{24}$ Thompson, K. W., "Time-dependent boundary conditions for hyperbolic systems," J. Comput. Phys., Vol. 68, 1987, pp. $1-24$

${ }^{25}$ Herbert, T., "Parabolized Stability Equations," Tech. Rep. AGARD-R-793, Von Karman Institute, 1994.

${ }^{26}$ Bridges, J. and Wernet, M., "Measurements of the aeroacoustic sound source in hot jets," AIAA Paper 2003-3130, 2003.

${ }^{27}$ Bridges, J. and Brown, C. A., "Parametric testing of chevrons on single flow hot jets," AIAA Paper 2004-2824, 2004.

${ }^{28}$ Tam, C. K. W. and Burton, D. E., "Sound generated by instability waves of supersonic flows. Part 1, two-dimensional mixing layers; Part 2, axisymmetric jets." J. Fluid Mech., Vol. 138, 1984, pp. 249-295.

${ }^{29}$ Tanna, H. K., "An Experimental Study of Jet Noise Part 1: Turbulent Mixing Noise," J. Sound Vibration, Vol. 50, 1977, pp. $405-428$.

${ }^{30}$ Freund, J., "Noise sources in a low-Reynolds-number turbulent jet at Mach 0.9," J. Fluid Mech., Vol. 438, 2001, pp. 277305.

${ }^{31}$ Crighton, D. G. and Huerre, P., "Shear-Layer Pressure-Fluctuations and Superdirective Acoustics Sources," J. Fluid Mech., Vol. 220, 1990, pp. 355-368.

${ }^{32}$ Tinney, C. E. and Jordan, P., "The near pressure field of co-axial subsonic jets," J. Fluid Mech., Vol. 611, 2008, pp. 175204 .

${ }^{33}$ Arndt, R. E. A., Long, D. F., and Glauser, M. N., "The proper orthogonal decomposition of pressure fluctuations surrounding a turbulent jet," J. Fluid Mech., Vol. 340, 1997, pp. 1-33.

${ }^{34}$ Reba, R., Narayanan, S., and Colonius, T., "Wave-packet models for large-scale mixing noise," Int. J. Aeroacoustics, To appear. 